\title{
Gastos hospitalarios en pacientes con fascioliasis en fase aguda, hidatidosis de tratamiento quirúrgico y neurocisticercosis en un hospital general en Chile
}

\author{
Alberto Fica y Thomas Weitzel
}

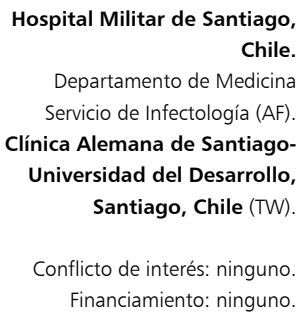

Recibido: 27 de noviembre de 2013 Aceptado: 7 de abril de 2014

Correspondencia a: Alberto Fica Cubillos albertofica@gmail.com

\author{
Hospital care expenses caused by acute fascioliasis, cystic echinococcosis, and \\ neurocysticercosis in Santiago, Chile
}

Background: Acute fascioliasis (FA), cystic echinococcosis (CE) and neurocysticercosis (NCC) are three endemic parasitic diseases in Chile for whom there is scarce information about the economic impact they represent during management at the hospital. Aims: To quantify and compare hospital care expenses caused by these three endemic helminth infections in a Chilean hospital. Methods: Retrospective analysis of hospital costs at a referral hospital in Santiago between 2006 and 2010. Hospital databases were used to identify patients with the corresponding infections, and those with sufficient data on hospital costs were included. Results: A total of 16 patients representing 21 cases were identified and analyzed: four with AF, eleven with CE, and six with NCC. Median hospital expenses for cases with AF were US\$ 1799 and mainly caused by bed-day costs. Median hospital costs for cases of CE were US\$ 4707 and the most important costs components were medications, bed-day costs and consumables. NCC patients had median costs of US\$1293, which were mainly due to bed-day costs. Non-parenchymatous or mixed forms of NCC showed a trend toward higher hospital costs compared with parenchymatous forms. Conclusions: Although helminth infections in Chile, an upper middle income country, are declining and considered rare in routine clinical practice, hospital care expenses caused by patients with AF, CE, and NCC are high and might still present an important economic burden to the Chilean healthcare system.

Key words: Fascioliasis, neurocysticercosis, cystic echinococcosis, hydatid disease, hospital costs, health expenditures.

Palabras clave: Fascioliasis, neurocisticercosis, equinococosis, hidatidosis, gastos de hospitalización, gastos en salud.

\section{Introducción}

L a fascioliasis en fase aguda (FA), la equinococosis o hidatidosis $(\mathrm{H})$ y la neurocisticercosis $(\mathrm{NCC})$ son enfermedades parasitarias desatendidas, de amplia distribución geográfica, causadas por larvas parasitarias y/o parásitos adultos inmaduros. Afectan principalmente regiones en desarrollo o empobrecidas que tienen programas limitados de prevención y control, lo que determina una información epidemiológica limitada para ellas tres ${ }^{1-5}$. La fascioliasis y la equinococosis o hidatidosis hepática o pulmonar son infecciones zoonóticas que están asociadas con la interacción de los seres humanos con animales domésticos. La cisticercosis es adquirida por la ingestión de huevos de Taenia solium eliminados por portadores de la tenia, los que a su vez son infectados en un ciclo antropo-zoonótico que involucra cerdos como hospederos intermediarios. Las tres enfermedades son causadas por parásitos inmaduros, los que al migrar y crecer en el ser humano, son capaces de causar un daño tisular grave con una morbilidad y mortalidad significativas ${ }^{2,3,5}$.
Chile es un país de ingresos medios con 17.500.000 habitantes y un producto interno bruto per capita de US\$ 14.403 al año 2011. Al igual en que muchos otros países de ingresos medios, el impacto clínico y de salud pública de las enfermedades parasitarias se encuentra en declinación $\mathrm{y}$, por lo tanto, estos patógenos obtienen cada vez menos atención. A pesar de ello, varias infecciones por helmintos son capaces de causar manifestaciones graves y aún son consideradas endémicas en Chile.

La información sobre los costos asociados a la FA, $\mathrm{H}$ y NCC, tanto para el paciente como para el sistema de salud, es muy limitada y no se dispone de estudios comparativos. Esta información es necesaria para efectuar estudios de costo-efectividad de diferentes estrategias de prevención ${ }^{6}$. Los objetivos del presente estudio son analizar y comparar los gastos hospitalarios de estas infecciones en un ambiente de ingresos medios en Latinoamérica. Esta información ayudará a comprender el impacto económico de estas infecciones y podría servir de base para futuros estudios de costo-efectividad de diferentes estrategias preventivas. 


\section{Pacientes y Métodos}

\section{Pacientes y escenario}

El Hospital Militar de Santiago funciona como un centro de referencia nacional del personal militar activo, retirado o sus cargas y también atiende pacientes civiles no relacionados con el mundo militar. Para nuestro estudio se consideraron todos los pacientes que tuvieran suficiente información en las bases de datos del hospital y que fueron atendidos por alguna de estas condiciones entre los años 2006 y 2010.

Se incluyeron pacientes con los siguientes códigos CIE-10: B66.3 (fascioliasis), B67.0 (infección hepática por E. granulosus), B67.1 (infección pulmonar por E. granulosus) y B69.0 (cisticercosis del sistema nervioso central). Los pacientes con el diagnóstico de alta de fascioliasis (B66.3) sólo fueron incluidos si presentaron un cuadro de FA. Adicionalmente, algunos pacientes con hidatidosis o NCC fueron identificados a través de los registros de farmacia interna sobre prescripciones de albendazol. Si el mismo paciente requirió varios ingresos por la misma infección parasitaria pero en una diferente localización, estos ingresos fueron considerados como casos separados. De la misma manera, si hubo un reingreso por complicaciones, fue también considerado como caso aparte.

\section{Gastos hospitalarios}

Los gastos hospitalarios de las tres enfermedades analizadas fueron obtenidos de la Unidad de Bioestadística del hospital. Los gastos fueron agrupados por día-cama, fármacos, insumos, pruebas de laboratorio, estudio de imágenes, cirugía, prestaciones de otros profesionales de la salud (por ejemplo psiquiatría, fisioterapia) y otros gastos, los que incluyeron procedimientos endoscópicos y gastos no clasificables. Los gastos fueron actualizados a diciembre de 2010 usando la información de actualización de precios del Instituto Nacional de Estadísticas (disponible en $\mathrm{http}$ ://encina.ine.cl/calculadoraipc) y expresados en US\$ de acuerdo a la paridad cambiaria oficial para entonces (1 US\$- 468,37 pesos chilenos).

\section{Análisis estadístico}

Las diferencias en gastos hospitalarios fueron comparados por una prueba no paramétrica (Sign test).

\section{Resultados}

Durante el período de estudio se identificaron 32 casos con FA, Ho NCC. Los detalles clínicos y epidemiológicos de estos pacientes han sido publicados previamente ${ }^{7-9}$. Se contó con información suficiente para 16 de estos casos, representando 21 ingresos: cuatro pacientes con FA (cuatro ingresos), siete pacientes con $\mathrm{H}$ (11 ingresos) y cinco pacientes con NCC (seis ingresos). De los pacientes con NCC, tres tenían una forma parenquimatosa y dos, formas no parenquimatosas o mixtas. La mediana de edad de los 16 pacientes fue 28 años (rango 3 a 70 años) y $69 \%$ eran de sexo masculino. La mediana de la estadía hospitalaria fue de 11 días (dos a 38 días). Todos los casos de FA fueron tratados medicamente mientras que todos los casos de hidatidosis y uno de NCC requirieron tratamiento quirúrgico. Los reingresos por complicaciones ocurrieron en dos casos de hidatidosis y uno con NCC. En la Tabla 1 se presentan mayores detalles.

\section{Gastos hospitalarios}

La mediana de los gastos de hospitalización para pacientes con FA, H y NCC alcanzó a US\$ 1.799 , US\$ 4.707, y US\$ 1.293, respectivamente (Tabla 2). Para pacientes con FA, el componente más importante fue la estadía hospitalaria ( $42 \%$ del total), seguido por el estudio de imágenes y medicamentos ( $17 \%$ cada uno). La mediana del gasto hospitalario diario fue US\$ 145.

Los casos con hidatidosis tuvieron valores más altos y los gastos se distribuyeron no sólo en cirugía (17,1\%), sino que también en medicamentos $(25,7 \%)$, días camas $(25 \%)$ e insumos $(21,3 \%)$. La mediana de gastos para pacientes con hidatidosis hepática y pulmonar fue de US\$ 5.019 (rango 1.766 a 6.259 ) y US\$ 4.015 (rango 1.363 a 7.627), respectivamente. Estas diferencias no fueron significativas. La mediana del gasto diario para estas dos formas de hidatidosis fue también similar (US\$ 449 vs US\$ 387) (Figura 1).

Los gastos por NCC fueron principalmente resultado del costo del día camas $(52,5 \%)$ seguido por gastos en cirugía $(12,8 \%)$, medicamentos $(12,6 \%)$ y estudio de imágenes $(10,2 \%)$. Hubo una tendencia no significativa a un mayor gasto en las formas extra-parenquimatosas o mixtas de NCC respecto a las formas parenquimatosas (mediana en gastos hospitalarios de US\$ 6.165 vs US\$1.293). Esta misma tendencia se observó en el gasto hospitalario diario (US\$ 262 vs US\$ 113) (Figura 1).

\begin{tabular}{|c|c|c|c|}
\hline & Fasciolasis aguda & Hidatidosis & Neurocisticercosis \\
\hline Pacientes/ingresos $\mathrm{n}$ & $4 / 4$ & $7 / 11$ & $5 / 6$ \\
\hline Mediana edad (rango) & 53,5 años (23-56) & 28 años (7-70) & 25 años (3-63) \\
\hline $\operatorname{Sexo}(F / M) n$ & $1 / 3$ & $1 / 6$ & $3 / 2$ \\
\hline Pacientes con ambiente rural $n$ & $0 / 4$ & $6 / 7$ & $5 / 5$ \\
\hline Mediana estadía hospitalaria (rango) & 14 días (7-21) & 10 días $(6-20)$ & 17 días (2-38) \\
\hline Procedimientos quirúrgicos $n$ & 0 & $\begin{array}{c}9 \text { ingresos } \\
\text { (resección quística) }\end{array}$ & $\begin{array}{c}1 \text { ingreso } \\
\text { (neurocirugía) }\end{array}$ \\
\hline Reingresos por complicaciones $n$ & 0 & 2 ingresos & 1 ingreso \\
\hline Desenlace (favorable/fatal) & $4 / 0$ & $7 / 0$ & $4 / 1$ \\
\hline
\end{tabular}


Tabla 2. Gastos hospitalarios (US\$) por ingresos por fascioliasis aguda, equinococosis o hidatidosis y neurocisticercosis. Hospital Militar, Santiago, Chile, 2006-2010

\begin{tabular}{|c|c|c|c|c|c|c|}
\hline \multirow{2}{*}{$\begin{array}{l}\text { Componente } \\
\text { Gastos totales }\end{array}$} & \multicolumn{2}{|c|}{$\begin{array}{l}\text { Fascioliasis aguda } \\
\qquad(n: 4)\end{array}$} & \multicolumn{2}{|l|}{$\begin{array}{l}\text { Hidatidosis } \\
\text { (n: 11) }\end{array}$} & \multicolumn{2}{|c|}{$\begin{array}{l}\text { Neurocisticercosis } \\
\text { (n: 6) }\end{array}$} \\
\hline & & $\%$ & & $\%$ & & $\%$ \\
\hline Día-cama & 3.429 & 42,0 & 12.044 & 25,0 & 10.880 & 52,5 \\
\hline Laboratorio & 830 & 10,0 & 1.698 & 3,5 & 960 & 4,6 \\
\hline Imágenes & 1.444 & 18,0 & 1.556 & 3,2 & 2.124 & 10,2 \\
\hline Medicamentos & 1.413 & 17,0 & 12.381 & 25,7 & 2.607 & 12,6 \\
\hline Insumos & 404 & 5,0 & 10.263 & 21,3 & 1.157 & 5,6 \\
\hline Evaluaciones por otros profesionales & 144 & 2,0 & 1.264 & 2,6 & 181 & 0,9 \\
\hline Cirugía & 0 & 0 & 8.259 & 17,1 & 2.658 & 12,8 \\
\hline Otros & 511 & 6,0 & 735 & 1,5 & 172 & 0,8 \\
\hline Total & 8.175 & 100 & 48.200 & 100 & 20.739 & 100 \\
\hline \multicolumn{7}{|c|}{ Mediana de gastos por paciente (rango) y \% del total } \\
\hline Día-cama & $728(568-1.406)$ & & $835(641-2.150)$ & & $587(81-7.824)$ & \\
\hline Laboratorio & $206(177-241)$ & & $133(0-358)$ & & $99(0-515)$ & \\
\hline Imágenes & $321(209-592)$ & & $69(0-385)$ & & $332(64-893)$ & \\
\hline Medicamentos & $203(121-885)$ & & $1.136(170-2.983)$ & & $191(0-1.742)$ & \\
\hline Insumos & $101(13-187)$ & & $747(64-2.312)$ & & $87(8-830)$ & \\
\hline Evaluaciones por otros profesionales & $30(0-84)$ & & $117(0-247)$ & & $23(0-71)$ & \\
\hline Cirugía & 0 & & $729(0-1.927)$ & & $0(0-2.658)$ & \\
\hline Otros & $103(7-298)$ & & $17(0-398)$ & & $26(0-72)$ & \\
\hline Total & $1.799(1.236-3.340)$ & & $4.707(1363-7.627)$ & & $1.293(425-11.906)$ & \\
\hline Mediana gastos (rango) por paciente-día & $145(112-198)$ & & 449 (129-894) & & $126(31-313)$ & \\
\hline
\end{tabular}

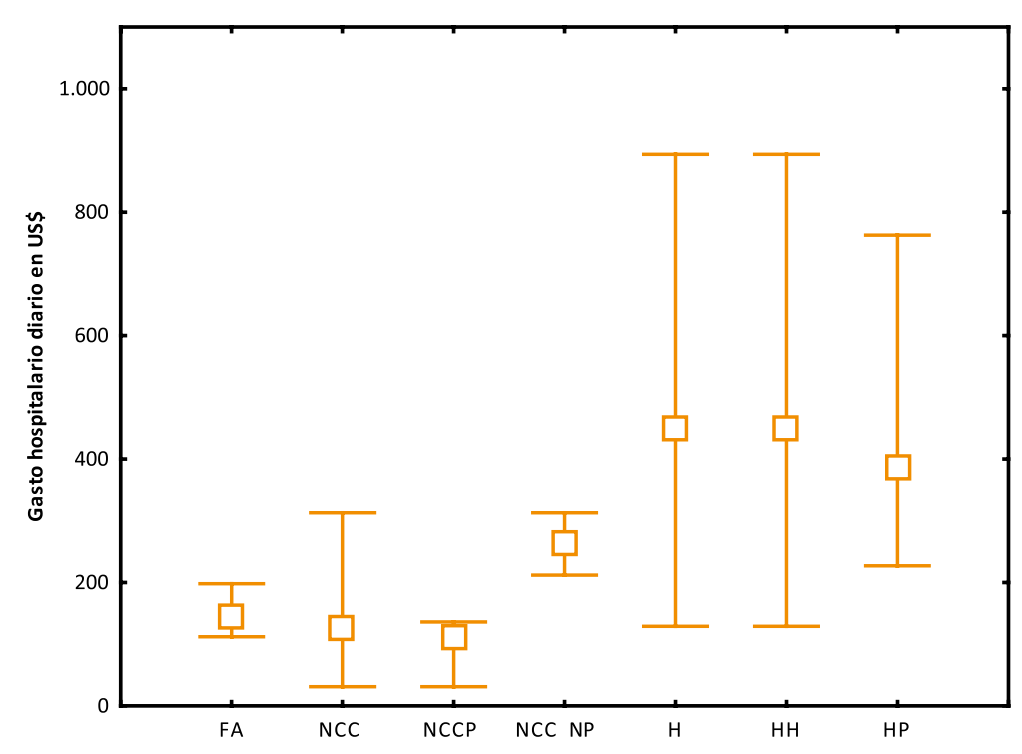

Figura 1. Diagrama de gastos hospitalarios diarios para pacientes con fascioliasis aguda (FA), diferentes formas de neurocisticercosis (NCC) y equinococosis o hidatidosis (H). Las líneas horizontales indican valores máximos y mínimos y los cuadrados indican la mediana. NCCP $=$ NCC parenquimatosa, NCC $\mathrm{NP}=\mathrm{NCC}$ no parenquimatosa o mixta, $\mathrm{HH}=$ Equinococosis hepática, $\mathrm{HP}=$ Equinococosis pulmonar.

\section{Discusión}

Las enfermedades parasitarias desatendidas tienen una amplia distribución y han sido en general poco estudiadas. Muchas de ellas representan enfermedades crónicas con un complejo impacto en las poblaciones afectadas. Algunas infecciones por helmintos, especialmente aquellas causadas por larvas o parásitos inmaduros, pueden ocasionar manifestaciones agudas y graves que demandan hospitalización y procedimientos médicos invasores. Tres enfermedades importantes de este grupo son endémicas en Chile: fascioliasis, hidatidosis por E. granulosus y neurocisticercosis.

Una parte relevante de la comprensión de la carga económica de estas infecciones es el análisis de los gastos hospitalarios en diferentes zonas geográficas al igual que la monitorización de los aumentos en los gastos médicos durante la atención de salud ${ }^{10}$. La falta de datos sobre estas enfermedades hace la estimación de los gastos a menudo difícil. Por ejemplo, un estudio efectuado en España, debió utilizar los antecedentes de Latinoamérica para estimar el impacto económico de la hidatidosis ${ }^{11}$.

Los gastos hospitalarios observados en nuestros casos con hidatidosis quística fueron similares a aquellos reportados por investigadores en Argentina (US\$ 3.965 a US\$ 
4.595 vs los US\$ 4.707 observado en nuestro trabajo) $)^{12,13}$ pero inferiores a los obtenidos en Uruguay (US\$ 6.721), ambos países sudamericanos de ingresos medios al igual que Chile ${ }^{14}$. En contraste, nuestros valores para pacientes con hidatidosis hepática (US\$ 1.766 a 6.259) fueron mayores a los números reportados en Chile en un hospital público entre 1990 y 1999 (US\$ 880 a 4.483) $)^{15}$ y muy superiores respecto a los registrados en una zona rural de China (US\$ 457 a 699) ${ }^{10}$. Por otro lado, nuestros gastos para pacientes con NCC fueron inferiores a aquellos reportados en el Condado de Los Ángeles en EUA (US\$ 37.000 vs US\$ 1.293 en este trabajo) ${ }^{16}$. Las diferencias comentadas parecen ser explicadas por múltiples factores incluyendo el valor del día cama, honorarios profesionales y diferencias en los precios de los procedimientos quirúrgicos y de los medicamentos. En pacientes con NCC, aquellos con formas parenquimatosas tuvieron una tendencia a un menor gasto diario en relación a los pacientes afectados por formas extra-parenquimatosas o mixtas (US\$ 113 vs US\$ 262 por día) lo que puede ser explicado por la necesidad de intervenciones quirúrgicas, reingresos y una mayor demanda de atención asociado a un peor pronóstico para las formas no parenquimatosas ${ }^{17}$. Los gastos en los casos de FA fueron explicados principalmente por la estadía hospitalaria. De acuerdo a nuestra experiencia, este componente depende de la experiencia del grupo médico tratante, puesto que en áreas de baja endemia esta enfermedad simula una condición maligna y el diagnóstico se retrasa ${ }^{7}$. No fue posible efectuar una comparación de los gastos asociados a FA ya que, de acuerdo a nuestro conocimiento, no existen publicaciones sobre esta condición que hayan evaluado este aspecto económico.

Nuestros datos subrayan la importante carga económica que tienen estas tres enfermedades parasitarias por caso hospitalizado teniendo como medianas valores entre US\$ 1.293 y 4.707. Este gasto es comparable o incluso superior que aquel observado en infecciones graves como meningitis causada por Haemophilus influenzae tipo b (US\$ 1.301 por caso) ${ }^{18}$ o absceso tubo-ovárico (US\$ 2.720 por caso $)^{19}$.

Aunque este trabajo comprende una pequeña cantidad de pacientes que provienen de un solo centro, permite una comparación entre las tres infecciones analizadas y una estimación de la magnitud de la carga económica que podrían generar estas infecciones parasitarias endémicas en el sistema de salud en Chile. Para tal efecto y tomando en cuenta que la fascioliasis y la NCC no son enfermedades notificables en Chile y que la hidatidosis está subnotificada $^{20}$, utilizamos la información proveniente del registro de los egresos hospitalarios para los años 2004 al 2008 por su disponibilidad, información gentilmente provista por el Departamento de Estadística e Información del Ministerio de Salud de Chile (DEIS). Durante el período
2004-2008 se registraron en promedio 917,2 egresos de hidatidosis y 150,1 de cisticercosis, pero sólo 2,8 casos de fascioliasis. La multiplicación de los gastos hospitalarios calculados en nuestro estudio (medianas) lleva a una estimación anual del gasto hospitalario de US\$ 4.317.260 para hidatidosis, US\$ 194.079 para NCC y US\$ 5.037 para FA en Chile. Como demuestra este cálculo, los gastos hospitalarios para estas tres infecciones podrían exceder los 4,5 millones de dólares al año. De esta estimación, el principal componente está dado por la hidatidosis (US\$ 4 millones) que de acuerdo a nuestro análisis y los egresos anuales, representa la condición más frecuente y la de mayores gastos (Tabla 2, Figura 1). Este número está en concordancia con una aproximación económica de la FAO para todo el cono sur latinoamericano, que indica un valor entre US\$ 14 a 30 millones por año para varios países de la región incluyendo Chile ${ }^{21}$. De una manera más amplia, los estudios que consideran la carga económica total asociada a hidatidosis (gastos por enfermedad y pérdida de ingresos por ausentismo) en Latinoamérica y la región del Caribe, estiman pérdidas monetarias por US\$ 48 millones y probablemente cerca de US\$ 120 millones por año ${ }^{22}$. El daño se incrementa al considerar los años perdidos por muerte prematura o discapacidad (DALYs) y las pérdidas en el ganado. Por ejemplo, la carga estimada de pérdidas humanas y animales en España alcanza a $€ 148$ millones por año ${ }^{11}$. En contraste, los pacientes que padecen de FA pueden tener un menor impacto para el sistema de salud debido a sus hospitalizaciones más cortas y menos procedimientos invasores o quirúrgicos. Estas estimaciones, sin embargo, deben ser tomadas como una estimación exploratoria debido al bajo número de casos, lo que requiere mayores estudios.

Como es evidente, este trabajo tiene varias limitaciones que incluyen su reducido número de casos pero la inclusión de unos pocos casos ha sido un rasgo común en estudios económicos de parasitosis desatendidas en Latinoamérica cuando estos estudios existen ${ }^{12,15}$. Por otra parte, los gastos hospitalarios fueron obtenidos en un solo hospital lo que podría cuestionar la validez de su estructura de cobro respecto a otras instituciones. Al respecto, se debe mencionar que los gastos en el Hospital Militar son generados por los mismos valores establecidos por el seguro público de salud en Chile y no representan por lo tanto una desviación a lo establecido legalmente para las instituciones públicas en todo el país. Tampoco este trabajo entrega información sobre gastos indirectos como ausentismo laboral o pérdida de ingresos laborales, gastos de transporte para controles médicos u otros gastos que aumentan el impacto económico de estas enfermedades. La mediana de edad para estas tres parasitosis presentadas en este trabajo, ubica a los pacientes en plena edad productiva lo que resalta el daño económico a la sociedad.

En conclusión, este trabajo demuestra que los gastos 
hospitalarios asociados al manejo de pacientes con FA, H y NCC son elevados y comparables a otras enfermedades infecciosas graves y que estas tres enfermedades parasitarias pueden representar una carga económica importante al sistema de salud en Chile.

\section{Resumen}

Antecedentes: La fascioliasis aguda (FA), la equinococosis o hidatidosis $(\mathrm{H})$ y la neurocisticercosis (NCC) son tres enfermedades parasitarias endémicas en Chile de las cuales hay escasa información sobre el impacto económico que ellas generan por atención hospitalaria. Objetivos: Cuantificar y comparar los gastos hospitalarios causados por estas tres infecciones por helmintos en un hospital en Chile. Materiales y Métodos: Análisis retrospectivo de gastos hospitalarios en un hospital de referencia en Santiago entre los años 2006 y 2010. Se incluyeron aquellos casos identificados en las bases de datos de hospital y que contaran con datos económicos suficientes. Resultados: Un total de 16 pacientes con 21 ingresos fue identificado y analizado: 4 con FA, 11 con $\mathrm{H}$ y 6 con NCC. La mediana de gastos hospitalarios para los ingresos por FA fue de US\$ 1.799, originados fundamentalmente por el costo del día cama. La mediana de los gastos en ingresos por $\mathrm{H}$ fue de US\$ 4.707 y los componentes más importantes en el gasto fueron los medicamentos, estadía hospitalaria e insumos. Los pacientes con NCC tuvieron una mediana de gastos de US\$ 1.293 explicado principalmente por la estadía hospitalaria. Los pacientes con formas no parenquimatosas o mixtas tuvieron una tendencia a presentar gastos más altos que aquellos con formas parenquimatosas de NCC. Conclusiones: Aunque las infecciones por helmintos están en declinación en Chile, un país de ingresos medios, y son consideradas raras en la práctica clínica, los gastos hospitalarios generados por la atención de pacientes con FA, H y NCC, son elevados y aún representan una importante carga económica para el sistema de salud chileno.

\section{Referencias bibliográficas}

1.- Marcos L A, Terashima A, Gotuzzo E. Update on hepatobiliary flukes: fasciolasis, opisthorchiasis and clonorchiasis. Curr Opin Infect Dis 2008; 21: 523-30.

2.- Keiser J, Utzinger J. Food-borne trematodiases. Clin Microbiol Rev 2009; 22: 466-83.

3.- World Organization for Animal Health. WHO/ OIE Manual on echinococcosis in humans and animals: a public health problem of global concern. Paris: OIE; 2001. Pp. 1-286 Available from: http://whqlibdoc.who.int/ publications/2001/929044522X.pdf (Último acceso 30 de noviembre de 2012).

4.- Moro P, Schantz P M. Cystic echinococcosis in the Americas. Parasitol Int 2006; 55 Suppl: S181-6.

5.- World Organization for Animal Health. WHO/ FAO/OIE Guidelines for the surveillance, prevention, and control of taeniosis/ cysticercosis. Paris: OIE; 2005. Pp. 1-156. Disponible en: http://www.oie.int/doc/ged/ d11245.pdf (Último acceso 30 de noviembre de 2012).

6.- World Health Organization. Research priorities for helminth infections: technical report of the TDR disease reference group on helminth infections. Technical report series; no. 972. Geneva: WHO; 2012. Pp 1-196. Disponible en: http://apps.who.int/iris/ bitstream/10665/75922/1/WHO_TRS_972_eng. pdf (Último acceso 22 de enero de 2013).

7.- Fica A, Dabanch J, Farías C, Castro M, Jercic M I, Weitzel T. Acute fascioliasisclinical and epidemiological features of four patients in Chile. Clin Microbiol Infect 2012; 18: 91-6.

8.- Fica A, Castro M, Soto A, Flores C, Oelker C,
Weitzel T. Neurocisticercosis, una enfermedad desatendida en Chile. Rev Chilena Infectol 2012; 29: 72-81

9.- Fica A, Soto A, Slater J, Peralta M, Humeres R, Castro $\mathrm{M}$, et al. Quince años de experiencia clínica con hidatidosis. Rev Chilena Infectol 2012; 29: 183-91.

10.- Yang Y R, Williams G M, Craig P S, McManus D P. Impact of increased economic burden due to human echinococcosis in an underdeveloped rural community of the People's Republic of China. PLoS Negl Trop Dis 2010; 4: e801.

11.- Benner C, Carabin H, Sánchez-Serrano L P, Budke C M, Carmena D. Analysis of the economic impact of cystic echinococcosis in Spain. Bull World Health Org 2010; 88 : 49-57.

12.- Larrieu E, Mercapide C, Del Carpio M, Salvitti J C, Costa M T, Romeo S, et al. Evaluación de las pérdidas producidas por hidatidosis y análisis del costo-beneficio de diferentes intervenciones estratégicas de control en la Provincia de Río Negro, Argentina. Bol Chil Parasitol 2000; 55: 8-13.

13.- Peláez V, Kugler C, Del Carpio M, Correa D, López E, Larrieu E, et al. Tratamiento de quistes hidatícos hepáticos por aspiración percutánea e inyección de solución salina hipertónica: Resultados de un trabajo cooperativo. Bol Chil Parasitol 1999; 54: 63-9.

14.- Torgerson P R, Carmona C, Bonifacino R. Estimating the economic effects of cystic echinococcosis: Uruguay, a developing country with upper-middle income. Ann Trop Med Parasitol 2000; 94: 703-13.

15.- Lorca M, Campanella C, Gravrilovics A, Lagos L, Florín C. Estimación de los costos totales y parciales de diagnóstico: tratamiento quirúrgico y seguimiento de la hidatidosis hepática en el Hospital San Juan de Dios Santiago Chile 1990-1999. Rev Chil Cir 2003; 55: 60-4.

16.- Croker C, Reporter R, Mascola L. Use of statewide hospital discharge data to evaluate the economic burden of neurocysticercosis in Los Angeles County (1991-2008). Am J Trop Med Hyg 2010; 83: 106-10.

17.- Takayanagui O M, Odashima N S. Clinical aspects of neurocysticercosis. Parasitol Int 2006; 55 (Suppl): S111-5.

18.- Levine O S, Ortiz E, Contreras R, Lagos R, Vial P, Misraji A, et al. Cost-benefit analysis for the use of Haemophilus influenzae type b conjugate vaccine in Santiago, Chile. Am J Epidemiol 1993; 137: 1221-8.

19.- Ovalle A, Casanova A, Kakarieka E, de Jourdan F, Salgado K. Epidemiología, resultados clínicos y costos del tratamiento del absceso tuboovárico, en un hospital público de Santiago. Rev Chil Obstet Ginecol 2008; 73 . 374-80.

20.- Serra I, Araya C, Araneda J. Evolución epidemiológica de la hidatidosis humana en Chile, 1965-1988: impacto de programas ganaderos en las regiones XI y XII. Rev Med Chile 1983; 121: 343-9.

21.- Food and Agriculture Organization of the United Nations. Estimación del impacto económico de la equinococosis quística en el Cono Sur (Argentina, Brasil, Chile y Uruguay). Rome: FAO; 2007. Pp. 1-20. Disponible en: http:// www.paho.org/spanish/ad/dpc/vp/hidatidosisimpacto-econ-07-fao.pdf (Último acceso $30 \mathrm{de}$ noviembre de 2012).

22.- Budke C M, Deplazes P, Torgerson P R. Global socioeconomic impact of cystic echinococcosis. Emerg Infect Dis 2006; 12: 296-303. 